\title{
Phase-locked soliton pairs in a stretched-pulse fiber laser
}

\author{
Ph. Grelu, F. Belhache, and F. Gutty \\ Laboratoire de Physique de l'Université de Bourgogne, Unité Mixte de Recherche 5027, B.P. 47870, 21078 Dijon, France
}

J.-M. Soto-Crespo

Instituto de Optica, Consejo Superior de Investigaciones Científicas, Serrano 121, 28006 Madrid, Spain

Received December 8, 2001

\begin{abstract}
We report the experimental observation of stable pulse pairs with a $\pm \pi / 2$ phase difference in a passively mode-locked stretched-pulse fiber ring laser. In our setup the stabilization of interacting subpicosecond pulses is obtained with a large range of pulse separations, namely, from 2.7 to $10 \mathrm{ps,}$, without the need for external control. (C) 2002 Optical Society of America

OCIS codes: $060.5530,140.3510$.
\end{abstract}

With the aim of developing compact high-repetitionrate sources for telecommunications applications, researchers have studied erbium-doped fiber lasers intensely for a decade. ${ }^{1}$ The field confinement in doped optical fibers provides both large optical gain and Kerr nonlinearity, key ingredients for passive mode locking. Anomalous dispersion leads to soliton pulse shaping and favors multiple pulsing with the increase of pumping power, as was observed in early experiments with passively mode-locked fiber laser cavities. It was then noticed that a slight change of the mode-locking conditions could group pulses into a more or less tight bunch instead of spacing them equally along the cavity. ${ }^{2,3}$ Recently, pulse bunching regained some attention because of the high stability of the pattern obtained under certain conditions, ${ }^{4-6}$ leading to new studies of the interactions between pulses in a fiber laser cavity.

Within the framework of the nonlinear Schrödinger equation, soliton-soliton interaction does not usually lead to fixing of pulse separation, except in the case of a train of solitons in quadrature, which is, nevertheless, not immune to perturbation. ${ }^{7}$ However, the increased complexity of the physical system, when either gain plus losses or higher-order dispersion terms are taken into account, allows one to find stable bound states of pulse pairs. On the one hand, modeling distributed gain and losses leads to the complex Ginzburg-Landau equation in which stable two-soliton bound states were predicted. ${ }^{8,9}$ An important feature of the stable bound states reported in Ref. 9 is the $\pm \pi / 2$ phase difference between the two pulses. On the other hand, the inclusion of large third-order dispersion was also shown to lead, in some cases, to the formation of bound multisoliton solutions, ${ }^{10}$ without any preferred phase difference between the pulses. The latter result could be relevant in balanced dispersion management, namely, with very close to zero path-averaged group-velocity dispersion and with the use of short sub-100-fs pulses, as was investigated with a $\mathrm{Cr}^{4+}$ :forsterite laser. ${ }^{11}$ Very recently, the observation of a closely interacting pair of pulses that formed a soliton bound state in an anomalous fiber ring was reported. ${ }^{6}$ To overcome timing jitter and achieve a bound state required injection of a strong cw laser field, and two possible separations between the two 310 -fs interacting pulses were observed, namely, 1.16 and $2.28 \mathrm{ps}$.

In this Letter we report, for the first time to our knowledge, that a pulse pair locks with the precise $\pi / 2$ phase relationship predicted within the framework of Ginzburg-Landau equation stability analysis. ${ }^{9}$ In addition, and contrary to the requirements mentioned in Ref. 6, no external injection was needed in our fiber cavity configuration, and pairs of interacting pulses were found to phase lock spontaneously with a large range of possible separations.

The fiber laser setup, a stretched-pulse fiber ring cavity comprising an open-air section, ${ }^{12,13}$ is illustrated in Fig. 1. The optical gain is provided by a 1.6-m-long 1400 -parts-in- $10^{6}$ (ppm) erbium-doped fiber with normal dispersion $[D=-40(\mathrm{ps} / \mathrm{nm}) / \mathrm{km}]$. The erbiumdoped fiber is followed by a polarization-insensitive coupler-isolator into which the 980-nm pumping light is injected. A 4.8-m length of SMF-28 fiber provides anomalous dispersion $[D=+17(\mathrm{ps} / \mathrm{nm}) / \mathrm{km}]$. Nonlinear polarization rotation that takes place along the propagation in both fibers makes transmission through polarizer P1 intensity dependent, allowing for passive mode locking to be triggered by an appropriate adjustment of the preceding wave plates. In pulse operation, however, it is necessary that

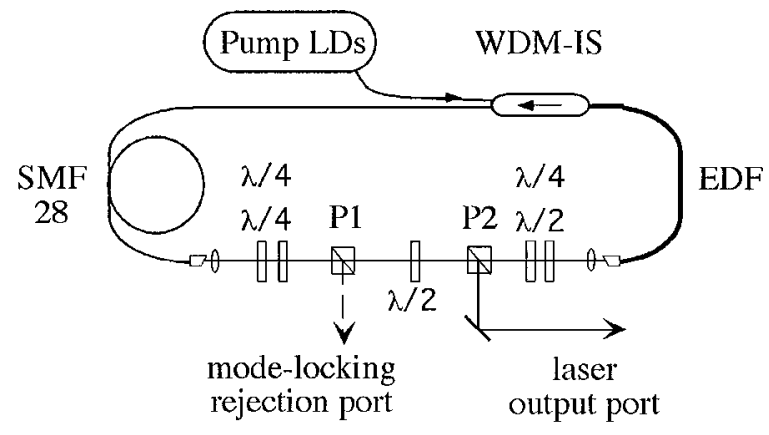

Fig. 1. Schematic of the fiber ring laser cavity pumped by laser diodes (LDs). WDM-IS, polarization-insensitive coupler-isolator; EDF, erbium-doped fiber; $\lambda / 4$, quarterwave plate; $\lambda / 2$, half-wave plate. 
P1 reject an important fraction $(30-70 \%)$ of the intracavity power for stabilization. Low-pedestal pulses may then be obtained at the output port, P2. Temporal characterization of the output intensity is performed with a laboratory-made optical autocorrelator that uses a 1-mm-thick $\beta$-barium borate crystal in type I second-harmonic generation and can be set in interferometric or noninterferometric mode. For spectrum analysis, we use a commercial high-resolution monochromator (Jobin-Yvon HR 640).

The laser self-mode-locks for a pumping power of more than $35 \mathrm{~mW}$. Operating with a slightly anomalous path-averaged dispersion of $\sim+3(\mathrm{ps} / \mathrm{nm}) / \mathrm{km}$, we observe low-pedestal sech-profiled pulses, whose FWHM duration varies from 400 to $650 \mathrm{fs}$. Stable bunches of pulses are easily obtained when the pump power is increased to more than $80 \mathrm{~mW}$, as described in Ref. 4. As many as five identical pulses of $\sim 15$-pJ power and 450-fs duration each are observed to bunch with a regular spacing of typically $20 \mathrm{ps}$, for a pump power of $\sim 250 \mathrm{~mW}$. We also noticed a very low level of timing jitter from the autocorrelation recordings as well as the presence of interference fringes in the spectrum, indicating phase locking. The procedure for obtaining a pair of interacting pulses is usually as follows: We start with a bunch of regularly spaced pulses whose separation is $\sim 20 \mathrm{ps}$, as this is the easiest stable configuration to be obtained in our setup. We then reduce the pump power to keep only two interacting pulses of the same amplitude, with 20-ps separation. Notice the presence of interference fringes in the spectrum, indicating that the two pulses are phase locked. However, the precise phase relationship between the two pulses is difficult to measure because of the large pulse separation. Reducing the pump power further below $50 \mathrm{~mW}$, we observe that the pair separation is decreased. We remark that the configuration of pulses undergoes some hysteresis with respect to the pumping power: We can keep a given configuration of pulses for a pump power significantly lower than the power required for formation of that given configuration. When the pump power is lowered, the decrease in the pair separation is not uniform but undergoes some jumps. These jumps are probably related to the high stability of the operation regime, in which a pulse pair remains precisely phase locked. Indeed, at the beginning, the pump power leads to a continuous decrease of the amplitude of both pulses, whereas the separation is kept constant and the pulse pair remains phase locked. Lowering the pump power further, we find that an abrupt transition happens in which the pulses get closer and stabilize with phase locking again at a fixed separation. The same process can be repeated two or three times.

When the pair separation is less than $10 \mathrm{ps}$, which is $\sim 18$ pulse widths, the phase relationship between the two phase-locked pulses can be inferred with good precision from the position of fringes in the pair spectrum. Figure 2(a) shows the spectrum of a pair of interacting pulses, whose asymmetry indicates the phase relation between pulses is neither 0 nor $\pi$. This spectrum is well fitted by the spectrum resulting from two 610-fs FWHM sech-profiled pulses separated by $6.8 \mathrm{ps}$ and with a phase difference of $\pi / 2$. The 610 -fs pulse width was deducted from the autocorrelation trace, and the time-bandwidth product of $0.32 \pm 0.01$ is compatible with unchirped sech-profiled pulses. Another proof of phase locking is obtained with the optical autocorrelation set in the interferometric mode. The resulting trace is shown in Fig. 2(b), and the presence of lateral peaks confirms the pulse separation as well as the equal amplitude of the pulses. Moreover, the fact that the lateral peaks comprise fringes, as shown in the insets, proves the stable phase relationship between the two pulses. The autocorrelation traces have been normalized so that average recorded intensity of the second-harmonic generation is unity where the pulses do not overlap. With this normalization, and when the pulses have identical amplitude, the fringes of the lateral peaks are expected to extend from 0.5 to 4.5 .

Another spectrum, recorded at a lower pump power of $29 \mathrm{~mW}$, is displayed in Fig. 3(a) and fitted by the spectrum of two pulses separated from $2.7 \mathrm{ps}$ and having a $-\pi / 2$ phase relationship. The corresponding interferometric autocorrelation function is shown on Fig. 3(b). For the experimental conditions of Figs. 2
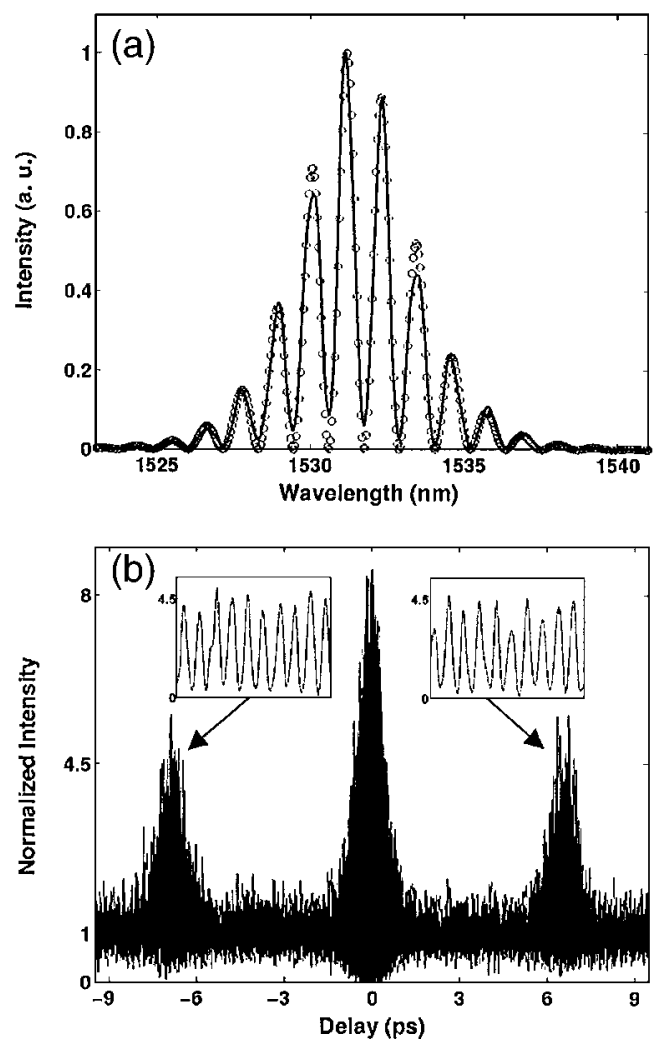

Fig. 2. (a) Spectrum of the laser output port (solid curve) fitted (open circles) by the spectrum of two unchirped 610 -fs sech-profiled pulses separated by $6.8 \mathrm{ps}$ and with a $\pi / 2$ phase difference. The pump power is $31 \mathrm{~mW}$. (b) Interferometric autocorrelation of the same output. Although experimental noise is visible, interference fringes appear clearly in the lateral peaks, as seen from the insets. This result indicates that the two pulses have a fixed phase relationship. 

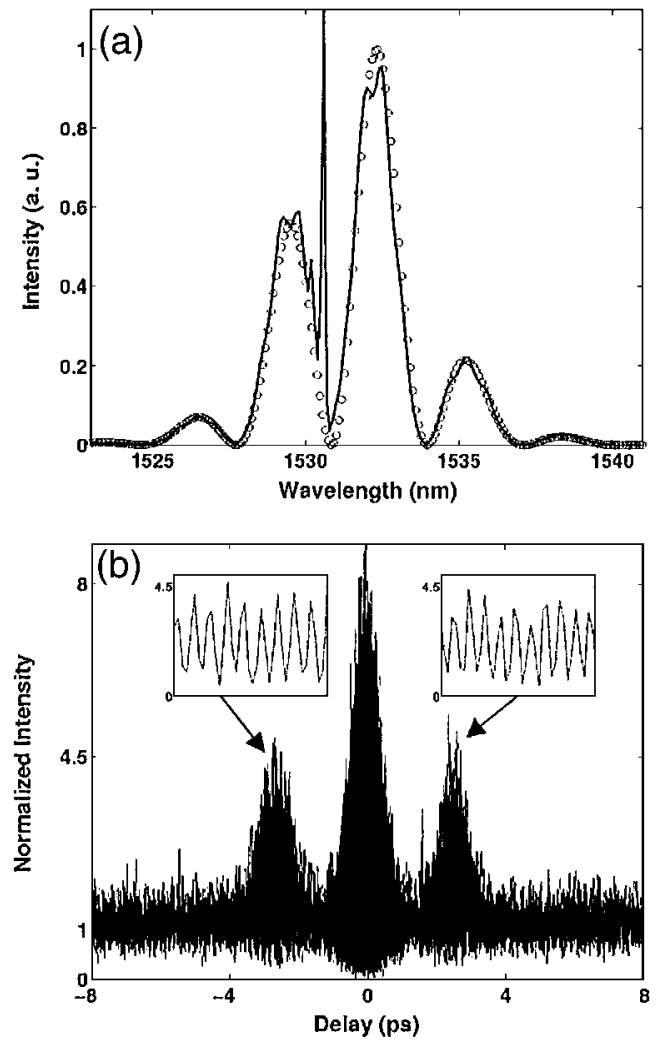

Fig. 3. (a) Spectrum of a closer pulse pair (solid curve) fitted (open circles) by the spectrum of two unchirped 540-fs sech-profiled pulses separated by $2.7 \mathrm{ps}$ and with a $-\pi / 2$ phase difference. The pump power is $29 \mathrm{~mW}$. (b) Corresponding interferometric autocorrelation trace.

and 3, there was no significant difference between the spectra taken at laser port P2 and the spectra taken at rejection port $\mathrm{P} 1$. This was always the case for low-energy pulse pairs. Output powers were approximately $0.8 \mathrm{~mW}$ at port $\mathrm{P} 1$ and $0.2 \mathrm{~mW}$ at port $\mathrm{P} 2$.

The $\pm \pi / 2$ phase relationship calculated from the previous spectra was predicted in a stability analysis of the complex Ginzburg-Landau equation. ${ }^{9}$ It is highly remarkable that this relation holds for pulses separated by more than 10 pulse widths in a highly perturbed dynamic system such as a stretched-pulse fiber laser. Finally, we also observed in our setup more-complicated structures of bunches comprising more than two phase-locked pulses that will be described in a future paper.

In conclusion, we have demonstrated experimentally that a passively mode-locked stretched-pulse fiber laser can support $\pm \pi / 2$ phase-locked pulse pairs. Depending on the pump power and mode-locking conditions, the pulse separation can be changed in a large range. The $\pm \pi / 2$ phase separation could be due to soliton-soliton interaction, as was theoretically predicted on the basis of the Ginzburg-Landau equation model. Because of the large range of pulse-to-pulse separations observed, we believe that incoherent effects such as gain recovery time $\mathrm{e}^{14}$ and electrostriction ${ }^{15}$ could fix the approximate separation, in particular when the pulses are far apart, whereas soliton-soliton interactions could be responsible for precise phase locking of pulses.

$\mathrm{Ph}$. Grelu gratefully acknowledges stimulating discussions with M. Haeltermann. The work of J.-M. Soto-Crespo was supported by the Dirección Generale de Enseñanza Superior under contracts BFM2000-0806 and CAM 07T/0063/2000. $\mathrm{Ph}$. Grelu's e-mail address is Phillippe.Grelu@ u-bourgogne.fr.

\section{References}

1. M. J. F. Digonnet, ed., Rare-Earth-Doped Fiber Lasers and Amplifiers, 2nd ed. (Marcel Dekker, New York, 2001).

2. D. J. Richardson, R. I. Laming, D. N. Payne, M. W. Phillips, and V. J. Matsas, Electron. Lett. 25, 730 (1991).

3. M. J. Guy, D. U. Noske, and J. R. Taylor, Opt. Lett. 18, 1447 (1993).

4. N. H. Seong, D. Y. Kim, and S. K. Oh, Electron. Lett. 37, 157 (2001).

5. F. Gutty, Ph. Grelu, N. Huot, G. Vienne, and G. Millot, Electron. Lett. 37, 745 (2001).

6. D. Y. Tang, W. S. Man, H. Y. Tam, and P. D. Drummond, Phys. Rev. A 64, 33814 (2001).

7. N. N. Akhmediev and A. Ankiewicz, Opt. Lett. 19, 545 (1994).

8. B. A. Malomed, Phys. Rev. A 44, 6954 (1991).

9. N. N. Akhmediev, A. Ankiewicz, and J.-M. Soto-Crespo, J. Opt. Soc. Am. B 15, 515 (1998).

10. J. D. Ania-Castañon, P. Garcia-Fernandez, and J. M. Soto-Crespo, J. Opt. Soc. Am. B 18, 1252 (2001).

11. B. Chassagne, G. Jonusauskas, J. Oberlé, and C. Rullière, Opt. Commun. 150, 355 (1998).

12. K. Tamura, L. E. Nelson, H. A. Haus, and E. P. Ippen, Appl. Phys. Lett. 64, 149 (1994).

13. K. Tamura and M. Nakazawa, Appl. Phys. Lett. 67, 3691 (1995)

14. J. N. Kutz, B. C. Collings, K. Bergman, and W. H. Knox, IEEE J. Quantum Electron. 34, 1749 (1998).

15. A. N. Pilipetskii, E. A. Golovchenko, and C. R. Menyuk, Opt. Lett. 20, 907 (1995). 